\title{
Time-frequency Analysis of Multicomponent LFM signal based on Hough and Chirplet Transform
}

\author{
Xueqin Zhang ${ }^{1,}$, Ruolun Liu, ${ }^{2, *}$ \\ ${ }^{1}$ Shandong University, Weihai, China, 264209 \\ ${ }^{2}$ Shandong Universit, Weihai, China, 264209
}

\begin{abstract}
The Chirplet Transform (CT) is effective in the characterization of IF for mono-component linear-frequency-modulated signal. However, During the initialization process, using the peak of the timefrequency map of the short-time Fourier transform to fit the line is greatly affected by noise. For the multicomponent signals, it is more difficult to distinguish and fit different IF lines. Since the Hough is good at a common algorithm for the line detection, the ridge edge fitting is replaced by the Hough transform in this paper. The experiment results show significant improvement in the obtained time-frequency representation.
\end{abstract}

\section{Introduction}

The random signals include stationary signals and nonstationary signals, the study of stationary signals has been relatively mature. However, the natural random signals are mostly non-stationary signal. Because the statistical properties of nonstationary signals are related to time, therefore, non-stationary signals must be processed simultaneously in both the time domain and the frequency domain. Time-frequency(T-F) analysis methods are usually divided into three categories. The first one is the linear time-frequencydistribution(TFD), often given by Fourier transform, suan as the short-time fourier transform (STFT), Gabor transform and continuous wavelet transform (CWT). The second type is the quadratic TFD, such as the is Wigner-ville distribution (WVD). The third type is the parameterized TFD [1-3] developed in recent years.

Due to the restriction of the Heisenber - Gabor inequality, neither the STFT nor the CWT is able to achieve a fine resolution in both the time and frequency domains. The WVD is a kind of quadratic transform that can achieve a highly accurate estimation for noise-free signals. When a signal has a linear frequency law and constant amplitude, its WVD will reduce to a row of delta functions along the linear IF trajectory [4-5]. However, in the cases of noisy signals, the WVD peak based estimator would be biased, and it has been shown that the bias-to-variance tradeoff is inevitable for the estimated IF result. The variance caused by the noise follows a decreasing function of the lag window length.

The Chirplet Transform (CT)[1-2] is a typical parametric TFD, which is particularly designed for the analysis of chirp-like signals with linear IF law. However, in the initialization process of the CT, the estimation of the parameters is based on the peak of the STFT magnitude, so the parameter estimation results are greatly affected by the noise. For multi-component signals, it is difficult to distinguish and fit different IF lines. We put forward the application of Hough transform in the STFT magnitude spectrum to obtain the robust parameter estimation of each component, then a set of time-frequency images are emerged by postprocessing to finally get the high concentration TFD.

Section 2 introduces the principle of the $\mathrm{CT}$ and discusses the way to analysis the multicomponent linear frequency modulated (LFM) signal. Section 3 presents the details of Hough transform. In the section 4, the feasibility of the proposed method is proved by the experiments. Conclusion is given in the section 5 .

\section{The review of the CT}

\subsection{The definition of Chirplet Transform}

The $\mathrm{CT}$ of a signal $\mathrm{s}(\mathrm{t})$ is defined as

$$
C T_{s}\left(t_{0}, \omega, \alpha ; \sigma\right)=\int_{-\infty}^{\infty} z(t) \psi_{\left(t_{0}, \alpha, \sigma\right)}^{*}(t) \exp (-j \omega t) d t
$$

where $z(t)$ is the analytical signal of $s(t)$, generated by the Hilbert transform, i.e., $\mathrm{z}(\mathrm{t})=\mathrm{s}(\mathrm{t})+\mathrm{jH}[\mathrm{s}(\mathrm{t})]$ and is a complex window given by

$$
\psi_{\left(t_{0}, \alpha, \sigma\right)}(t)=w_{(\sigma)}\left(t-t_{0}\right) \exp \left(-j \frac{\alpha}{2}\left(t-t_{0}\right)^{2}\right)
$$

where to is the time shift and $\alpha$ is the chirp rate. $\omega$ is uauslly taken as Gaussian function expressed as

$$
\begin{aligned}
& w_{(\sigma)}(t)=\frac{1}{\sqrt{2 \sigma \sigma}} \exp \left(-\frac{1}{2}\left(\frac{t}{\sigma}\right)^{2}\right) \\
& \left\{\begin{array}{l}
z(t)=z(t) \Phi_{\alpha}^{R}(t) \Phi_{\alpha}^{M}\left(t, t_{0}\right) \\
\Phi_{\alpha}^{R}(t)=\exp \left(-j \alpha t^{2} / 2\right) \\
\Phi_{\alpha}^{M}\left(t, t_{0}\right)=\exp \left(j \alpha t_{0} t\right) \\
A\left(t_{0}\right)=\exp \left(-j t_{0}{ }^{2} \alpha / 2\right)
\end{array}\right.
\end{aligned}
$$

\footnotetext{
* Corresponding author: ruolun.liu@ @sdu.edu.cn
} 
From this definition, it can be seen that the CT can be decomposed into a series of operations: 1) rotating the signal under consideration by a angle of $\arctan (-\alpha)$ in the time-frequency plane; 2) shifting the signal by a frequency increment of $\alpha t_{0}$ and 3) applying STFT with the Gaussian window. This process can be depicted in the Figure 1. The solid line is the IF line of the target LFM signal. The dot-dashed line is the IF line after the rotation, and the dashed line represents the IF line after translation.

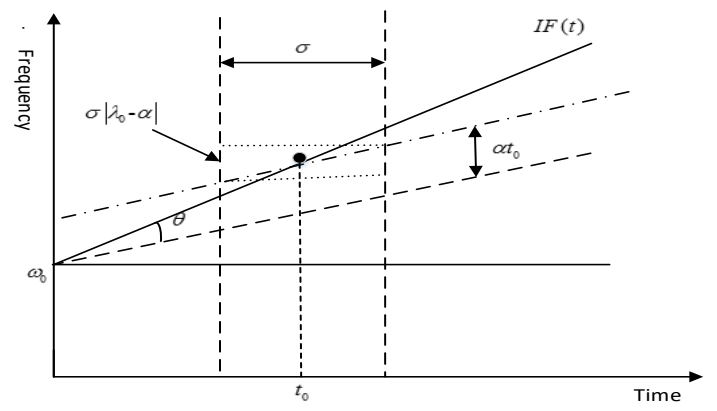

Figure 1. the Chirplet Transform

\subsection{Time-frequency analysis for mono- component LFM signal based on the CT}

As mentioned earlier, given a set of properly determined kernel characteristic parameters, the CT could produce a high-quality TFD for a considered signal. The result can have an excellent T-F concentration, and the IF trajectory can be easily identified. Therefore, the determination of proper parameters is critical for the application of the CT method. Briefly speaking, the basic idea of the CT based T-F analysis method is using the kernel characteristic parameters to form the TFD, then find the maximum value along time axis in the time-frequency plane. The resulting maximum line approximation is considered to be an IF trajectory. The chirp parameter obtained by the line fitting will be reapplied to the CT transformation. The procedure can be repeated until no evident improvement is observed in the T-F concentration of the TFD.

To measure the T-F concentration of the TFD, the Rényi entropy can be used with the definition:

$$
\operatorname{REN}(s)=-\iint \log \left|C T_{s}(t, \omega)\right|^{3} d t d \omega
$$

And the termination condition can be set as

$$
\xi_{(s)}=\left|R E N_{(i+1)}(s)-\operatorname{REN}_{(i)}(s)\right|<\delta
$$

$\mathrm{Or}$

$$
\xi_{(s)}=\frac{\left|\operatorname{REN}_{(i+1)}(s)-\operatorname{REN}_{(i)}(s)\right|}{\left|R E N_{(i+1)}(s)\right|}<\delta
$$

where $\delta$ is a predetermined threshold. If the parameters of the initialization are not accurate, it will lead to be a lower overall T-F concentration. Because STFT is susceptible to noise, the robustness of the whole algorithm is not strong. Fortunately, the FrFT can compensate for this shortcoming.

\subsection{T-F analysis for multicomponentLFM signal based on the $\mathrm{CT}$}

Taking the two-component signal as an example, it will give more accurate parameters through line fitting before applying them to CT. In this section, the difference of the TFD is obtained by the specific analogue signal analysis.A simulated multicomponent LFM signal is considered as

$$
s(t)=\sin \left(2 \pi\left(40 t-t^{2}\right)\right)+\sin \left(2 \pi\left(10 t+\frac{5}{4} t^{2}\right)\right) \quad(0 \leq t \leq 15 s)
$$

Its IF lines are $\mathrm{f} 1(\mathrm{t})=40-2 \mathrm{t}$ and $\mathrm{f} 2(\mathrm{t})=10+2.5 \mathrm{t}$. So the actual parameters should be $\alpha_{1}=-4 \pi \quad \alpha_{1}^{\prime}=5 \pi$. Suppose we have these two exact parameters, and then apply them into the CT transformation and observe the properties of the TFD graphs.

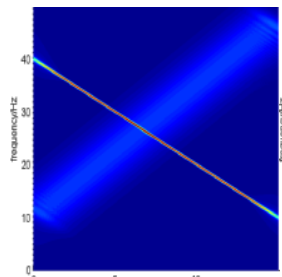

(a)

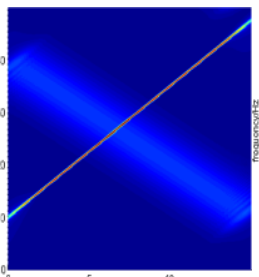

(b)

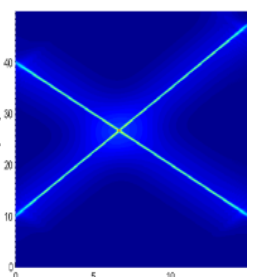

(c)
Figure 2. The TFD results of $\mathrm{s}(\mathrm{t})$ using $\mathrm{CT}$ with parameter (a) $\alpha_{1}=-4 \pi$, (b) $\alpha_{1}^{\prime}=5 \pi$ (c) the superposition of them

From the Figure 2, one can see that when the parameter $\alpha$ takes $\alpha 1$, the component with IF of $\mathrm{fl}(\mathrm{t})$ has good T-F concentration in the CT-TFD, while the component with IF of $\mathrm{f} 2(\mathrm{t})$ has very low $\mathrm{T}-\mathrm{F}$ concentration in the CT-TFD with also very low amplitude. And it shows the opposite situation when $\alpha$ takes $\alpha 2$. Add the two spectra together, a good TFD can be obtained as the following. Observing those TFDs above, if we filter out a very low percentage of components with a threshold before adding up the two spectra, we will get a sharper TFD with higher T-F concentration given by Figure 3 .

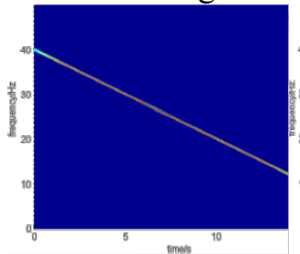

(a)

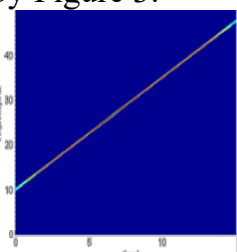

(b)

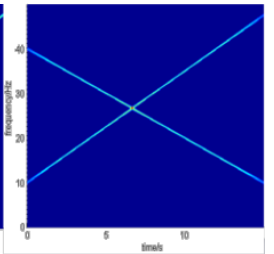

(c)
Figure 3. The results of the threshold filtering

\section{The review of Hough}

Through the description of the previous section, we know that as long as we can get the precise FM parameters, we can get the TFD with high T-F concentration. However, for multicomponent signal with low SNR, the difficulty will be signifcantly huge. Inspired by the refereence [7], we apply the robust Hough transform to detect the IF lines in the CT-TFD to depress the noise during the process of line fitting. The reason why we adopt this image processing technique is that the Hough transform can detect multiple lines accurately, even in the low SNR situations. 


\subsection{The principle of detecting straight lines of Hough transform}

For a straight line in the Cartesian coordinate plane, there are two common representations: point-slope form and two points form. In the Hough transformation, however, another representation is considered: coordinate $(r, \theta)$ Hough transformation, however, another representation is considered: coordinateis used to represent a straight line where $r$ is the distance from the straight line to the origin, $\theta$ is the angle between the $\mathrm{x}$ axis and the perpendicular line passing through the origin as shown in the Figure 4.

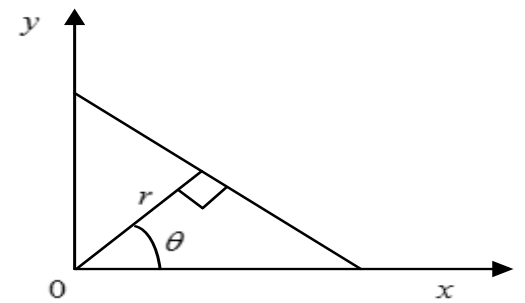

Figure 4. the line representation in Hough transform

The idea of using Hough transform to detect straight lines needs to assume $\mathrm{N}$ straight lines for each point, usually $\mathrm{N}=180$. In this paper, the angle accuracy of the detected line is 1 degree. The coordinates $(r, \theta)$ of the $\mathrm{N}$ lines are calculated respectively. If there are a total of $\mathrm{K}$ points to be checked, the final number of coordinates are $\mathrm{N} \times \mathrm{K}$. If a number of points are on the same line, then there must be a same number of points with the same the same $(r, \theta)$ coordinate. The following example shows that if there are three points in the plane, we can determine whether or not the three points are on the same straight line by their Hough coordinates.

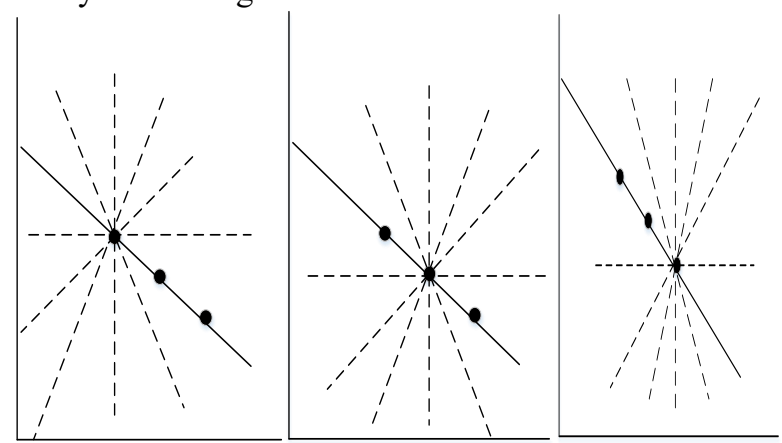

Figure 5. Six same straight lines oft three points

Table 1. The coordinates of lines in Figure 6

\begin{tabular}{|c|c|c|c|c|c|}
\hline Angle & Dist. & Angle & Dist. & Angl & Dist. \\
\hline 0 & 40 & 0 & 57.1 & 0 & 74.6 \\
30 & 69.6 & 30 & 79.5 & 30 & 89.6 \\
60 & 81.2 & 60 & 80.5 & 60 & 80.6 \\
90 & 70 & 90 & 60 & 90 & 50 \\
120 & 40.6 & 120 & 23.4 & 120 & 6.0 \\
150 & 0.4 & 150 & -19.5 & 150 & -39.6 \\
\hline
\end{tabular}

In the Table 1 , six line coordinates $(r, \theta)$ for each check point are calculated, and a total of 18 coordinates are obtained. It can be found that the three points are approximately on the same straight line of $(80.7,60)$.

\subsection{The result of straight line detection with Hough transform}

In order to highlight the advantages of Hough transform, the analog signal of equation (8) is generated at the SNR of $2 \mathrm{~dB}, 0 \mathrm{~dB}$, and $-2 \mathrm{~dB}$. Then the STFT and the corresponding Hough transform are calculated.

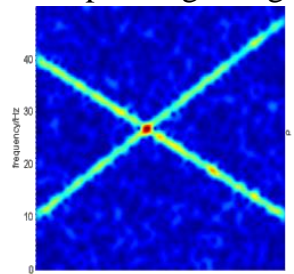

(a)

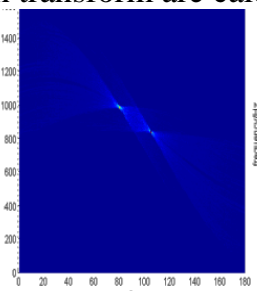

(b)

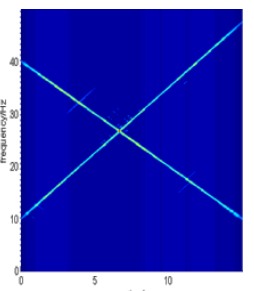

(c)
Figure 6. The STFT (a), and the corresponding Hough transform (b), and the TFD result of the proposed algorithm (c) of $s(t)$ at the SNR of $2 \mathrm{~dB}$

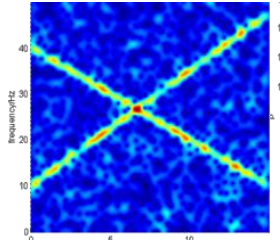

(a)

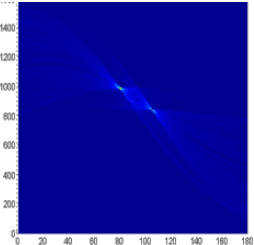

(b)

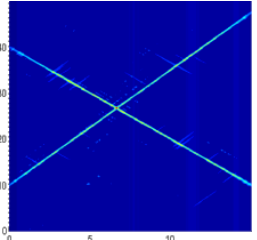

(c)
Figure 7. The STFT (a), and the corresponding Hough transform (b), and the TFD result of the proposed algorithm (c) of $s(t)$ at the SNR of $0 \mathrm{~dB}$

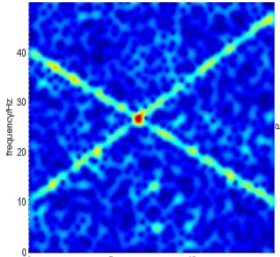

(a)

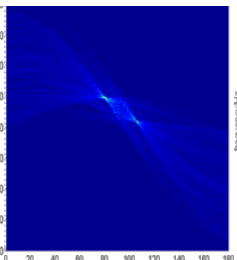

(b)

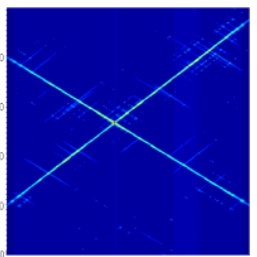

(c)
Figure 8. The STFT (a), and the corresponding Hough transform (b) , and the TFD result of the proposed algorithm (c) of $s(t)$ at the SNR of $-2 \mathrm{~dB}$

From the above results, one can see clearly that in the case of low SNR, the STFT is relatively fuzzy, and the ridge edge extraction alone will not give the exact results. However, the accuracy of Hough transform is relatively high at all the 3 SNR conditions. That is the reason why the Hough transform is adopted in the time-frequency analysis of multicomponent LFM signal in this paper.

\section{Validations}

In summary, the proposed algorithm is as follows. 
Table 2. The steps of the proposed algorithm

\begin{tabular}{l}
\hline Algorithm:Improved parameterized TFD \\
\hline Input: $\left(\alpha_{1}, \alpha_{2} \ldots \alpha_{N}\right)=(0,0, \ldots, 0), \delta, \sigma$ \\
Output:TFD image of multicomponent LFM signal \\
At the $k$-th step: \\
1)Use zero initials to calculate CT in order to obtain \\
$T F D(k)$; \\
to obtain $T F D(k)$; \\
2)Apply the Hough transform to the $T F D(k)$, and \\
calculate the FM parameters $\left(\alpha_{1}^{\prime}, \alpha_{2}^{\prime}, \ldots, \alpha_{n}^{\prime}\right) ;$ \\
3)Recalculate CT and the $\left\{T F D_{1}(k+1), \ldots\right.$, \\
$\left.T F D_{N}(k+1)\right\}$ with the new parameters; \\
4)Apply the threshold filtering to the $\left\{T F D_{1}(k+1), \ldots\right.$, \\
$\left.T F D_{N}(k+1)\right\}$ and then $T F D=\sum_{n=1}^{N} T F D_{n}(k+1)$ \\
5)Calculation termination criterion $\xi_{k}$ \\
6)If $\xi_{k}>\delta, k=k+1, T F D_{k}=T F D$, and go to the
\end{tabular}

In order to verify the proposed algorithm, the analog signals used in the previous experiment are used again. The results are compared with the STFT through Figure 6 to Figure 8.

\section{Conclusions}

The Chirplet transform has the advantage of high T-F centralization, but is easily affected by the noise. In addition, many nonstationary signals are of multicomponent. The decomposition of multicomponent signal [7-8] into single component signals under noise conditions is a difficult problem. For the multicomponent LFM signal, the Hough transform is adopted to the parametric T-F analysis to obtain the result with good concentration. However, for the multicomponent nonlinear FM signal, there is no effective trajectory detection method, so it will be more difficult to decompose each nonlinear FM component. That will be the focus of our future work.

\section{References}

1. S.Mann and S.Haykin, The chirplet transform: Physical considerations, IEEE Trans. Signal Process., vol. 43, no. 11, pp. 2745-2761, Nov. 1995.

2. Z. K. Peng, G. Meng, F. L. Chu, Z. Q. Lang, W. M. Zhang, and Y. Yang,"Polynomial chirplet transform with application to instantaneous frequency estimation," IEEE Trans. Instrum. Meas., vol. 60, no. 9, pp. 3222-3229, Sep. 2011.

3. Y. Yang, Z. K. Peng, G. Meng, and W. M. Zhang, "Spline-kernelledChirplet transform for the analysis of signals with time-varyingfrequency and its application," IEEE Trans. Ind. Electron., vol. 59, no. 3, pp. 1612-1621, Mar. 2012.

4. J. A. Rosero, L. Romeral, J. A. Ortega, and E. Rosero, "Short-circuitdetection by means of empirical mode decomposition and WignerVilledistribution for PMSM running under dynamic condition," IEEE Trans.Ind. Electron., vol. 56, no. 11, pp. 4534-4547, Nov. 2009.

5. V. Climente-Alarcon, J. A. Antonino-Daviu, M. Riera-Guasp, andM. Vlcek, "Induction motor diagnosis by advanced notch FIR filters andThe Wigner-Ville distribution," IEEE Trans. Ind. Electron., vol. 61, no.8,pp. 4217-4227, Aug. 2014.

6. Almeida L B. The fractional Fourier transform and time-frequencyrepresentations. IEEE Transactions on Signal Processing,1994, 42(11):3084-3091.

7. LJ. Stankovi, M. Dakovi, T. Thayaparan, V. PopoviBugarin, "Inverse Radon Transform Based MicroDoppler Analysis from a Reduced Set of Observations," IEEE Trans. on Aerospace and Electronic Systems, vol. 51, no. 2, pp.1155-1169, Apr. 2015.

8. M. Dakovi, LJ. Stankovi, "Estimation of sinusoidally modulated signal parameters based on the inverse Radon transform," ISPA 2013, pp. 302307, Trieste, Italy, 4-6 Sept. 2013. 